\title{
A NEW NON-ECLIPSING CV SDSSJ 122405.58+184102.7 - A PROBABLE MEMBER TO THE SW SEXTANTIS TYPE STARS
}

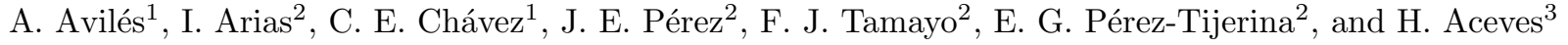 \\ Received May 14 2019; accepted October 232019
}

\begin{abstract}
We present observational evidence that helps classify the non-eclipsing binary system SDSSJ 122405.58+184102.7 as a new member of the SW Sextantis (SW Sex) class. First, from the analysis of the optical light curve, we identify the presence of two periodic signals that develop on different time scales. The first one is the orbital period of $0.167811(1)$ days $(=4.027464(3) \mathrm{h})$ and the second one is the white dwarf spin period of 28.6 minutes. This second period is probably the first evidence for the presence of a magnetic white dwarf in the system. The second evidence is the presence of the HeII $\lambda 4886$ emission line in its optical spectrum. In this work we interpret the detected periodicities within the context of a magnetic accretion model for SW Sex stars.
\end{abstract}

\section{RESUMEN}

Presentamos evidencia observacional que ayuda a clasificar al sistema binario no eclipsante SDSSJ 122405.58+184102.7 como un nuevo miembro de la clase SW Sextantis (SW Sex). Primero, a partir del análisis de la curva de luz en el óptico, identificamos la presencia de dos señales periódicas que se desarrollan en diferentes escalas de tiempo. La primera es el período orbital de 0.167811(1) días $(=4.027464(3) \mathrm{h})$ y la segunda es el período de giro de la enana blanca de $28.6 \mathrm{mi}-$ nutos. Este segundo período es probablemente la primera evidencia de la presencia de una enana blanca magnética en el sistema. La segunda evidencia es la presencia de la línea de emisión HeII $\lambda 4886$ en su espectro. En este trabajo interpretamos las periodicidades detectadas dentro del contexto de un modelo de acreción magnético para estrellas SW Sex.

Key Words: binaries: general - novae, cataclysmic variables - stars: individual: SDSSJ $122405.58+184102.7$

\section{INTRODUCTION}

Cataclysmic variables stars (CVs) are semi-detached binary systems composed by a white dwarf (WD) as primary star and a late-type main sequence secondary star. The later fills its Roche Lobe and transfers matter through the Lagrangian point $\mathrm{L}_{1}$ onto the WD via Roche-lobe overflow.

In non-magnetic systems, the incoming material forms an accretion disk around the WD (Warner 1995). This accretion disk periodically reaches a critical surface density value, leading to the development of thermal instabilities (Meyer \& Meyer-

\footnotetext{
${ }^{1}$ Facultad de Ingeniería Mecánica y Eléctrica, UANL, México.

${ }^{2}$ Facultad de Ciencias Físico Matemáticas, UANL, México.

${ }^{3}$ Instituto de Astronomía, Universidad Nacional Autónoma de México, México.
}

Hofmeister 1981), triggering an outburst that increases the system brightness by up to 8 magnitudes. The system returns to quiescence in a few days or weeks. This photometric behavior defines a CV class named Dwarf Nova (Patterson et al. 1981; Howell et al. 1995; Szkody \& Mattei 1984). Besides the light curve pattern method to classify CVs, there are other parameters that help to do so, such as the orbital period, the mass transfer rate or the magnetic field strength.

The orbital period defines the separation of the binary components and establishes the evolutionary status of the secondary star. CVs with periods over 3 hours contain secondaries stars with radiative cores, and as the orbital period decreases below 3 hours the companion reaches a mass low enough to become 
fully convective (Verbunt \& Zwaan 1981; Rappaport, Verbunt, \& Joss 1983; Paczynski \& Sienkiewicz 1983; Spruit \& Ritter 1983).

A high mass transfer rate defines the nova-like (NL) CV class. It helps to maintain a hot and stable accretion disk and prevents dwarf-nova-type outbursts. We refer to Warner (1995) for a general review on NLs and CVs in general, or Mizusawa et al. (2010); Balman et al. (2014) where they describe the UV and X-ray properties of several NL systems.

The strength of the magnetic field defines two types of CVs named Polars and Intermediate Polars (IP). In Polars, the magnetic field is so strong $(B \geq 20 \mathrm{MG})$ that it forces the WD to spin around its polar axis with the orbital period (Campbell 1997). When the incoming ionized accretion stream encounters the magnetosphere around the $\mathrm{WD}$, it is driven by the magnetic field lines, forming a shock region at the magnetic poles, rather than an accretion disk. If the magnetic field is weaker, as in IP CVs $(B \approx 2-8 \mathrm{MG})$, a truncated accretion disk may form outside the magnetosphere. The incoming material hits the magnetosphere at all points over the inner edge of the accretion disk, and the flow becomes an accretion curtain rather than a single converging stream (Rosen, Mason \& Cordova 1988; Hellier, Cropper \& Mason 1991). Another effect of a weaker magnetic field is that the WD rotates as fast as ten times the orbital frequency, so synchronous rotation is no longer present.

Within the CV zoo it is possible to find the SW Sex stars, which are a subclass of high accretion rate CVs; i.e. NL members. They were first identified by Thorstensen et al. (1991) as eclipsing NL CVs with an orbital period around 3 to 4 hours. Radial velocities studies reveal that the emission lines vary periodically; the Balmer series lines lagging behind the expected phase for a WD according to photometric ephemeris. Their spectra show single or doublepeaked emission lines, regardless of inclination angle. The single-peaked emission lines are thought to originate from the material encountering a magnetic accretion curtain close to the surface of the WD (Hoard et al. 2003). In contrast, the double-peaked profiles are likely a consequence of phase-dependent absorption components, as revealed by their trailed spectra.

Nowadays, SW Sex stars are thought to be the dominant population of CVs with periods within 3 and 4.5 hours (Rodríguez-Gil et al. 2007). A few cases of confirmed SW Sex stars show circular polarization, reflecting its magnetic nature (RodríguezGil et al. 2001). Additionally, photometric quasi- periodic oscillations (QPOs) are features observed recently in SW Sex stars. It is possible that this QPOs reflect the rotation of an underlying magnetic WD (Patterson et al. 2002). Finally, evidence has been mounting that the SW Sex phenomenon is an evolutionary stage in the evolutionary process of CVs (Schmidtobreick et al. 2012).

SDSS J1224 (12:24:05.58+18:41:02.7) is a relatively bright star with $g=16.01 \mathrm{mag}$. The presence of absorption and emission lines in its spectrum point to its being a pre-CV; a possible source for this could be the irradiation from the secondary by a hot WD (Szkody et al. 2011). Szkody et al. (2014) reported 126 min of time-resolved spectra taken in 2011, and concluded that there was no significant radial velocity variation during that time interval, so the system likely has a long orbital period or a low inclination angle.

In this work we present observational evidence that allows to classify SDSS J1224. This paper is organized as follows. In $\S 2$ we present our observations, and in $\S 3$ the data analysis and results. In $\S 4$ a discussion is provided with our main conclusions stated in $\S 5$.

\section{OBSERVATIONS AND DATA REDUCTION}

\subsection{Photometric Observations}

Differential time-resolved photometry of SDSSJ 1224 was conducted using the direct CCD imaging mode of a $0.84 \mathrm{~m}$ telescope, located at Observatorio Astronómico Nacional at the Sierra San Pedro Mártir $\left(\mathrm{OAN} \mathrm{SPM}^{4}\right)$ in México. We acquired a long series of photometric data in the $V$ broadband JohnsonCousins filter, with exposure times ranging from 30 to $60 \mathrm{~s}$. We observed the system on three nights in April 2016, four nights in March 2017 and three nights in April-May 2017 with the same instrument and configuration. Table 1 shows the log of these photometric observations.

Data reduction was performed using standard IRAF procedures (Tody 1986, 1993). The images were bias-corrected and flat-fielded before aperture photometry was carried out. We used photometric aperture radii of 2.0 times the PSF FWHM. The uncertainty in the differential photometry was estimated to be in the range 0.02 to 0.05 mag, according to the magnitude dispersion observed for the field stars. We used the star TYC 1445-830-1 in the field of view as the reference star.

${ }^{4}$ http://www.astrossp.unam.mx 
TABLE 1

\section{LOG OF TIME-RESOLVED OBSERVATIONS OF $S D S S J 1224$ IN THE $V$ BAND}

\begin{tabular}{lccc}
\hline Date & $\begin{array}{c}\text { HJD Start }+ \\
2457000\end{array}$ & $\begin{array}{c}\text { Exp. Time } \\
\text { Number of } \\
\text { Integrations }\end{array}$ & $\begin{array}{c}\text { Duration } \\
\mathrm{h}\end{array}$ \\
\hline 01 April 2016 & 480.73269 & $30 \mathrm{~s} \times 387$ & 3.2 \\
02 April 2016 & 481.64345 & $60 \mathrm{~s} \times 387$ & 6.5 \\
03 April 2016 & 482.67408 & $60 \mathrm{~s} \times 240$ & 4.0 \\
23 March 2017 & 836.729960 & $30 \mathrm{~s} \times 200$ & 1.7 \\
24 March 2017 & 837.682042 & $60 \mathrm{~s} \times 370$ & 6.2 \\
25 March 2017 & 838.667957 & $60 \mathrm{~s} \times 240$ & 4.0 \\
26 March 2017 & 839.645395 & $60 \mathrm{~s} \times 210$ & 3.5 \\
29 April 2017 & 872.667346 & $60 \mathrm{~s} \times 281$ & 4.7 \\
30 April 2017 & 873.660104 & $60 \mathrm{~s} \times 270$ & 4.5 \\
01 May 2017 & 874.650873 & $60 \mathrm{~s} \times 210$ & 4.0 \\
\hline
\end{tabular}

\subsection{Spectroscopic Observations}

We carried out spectroscopic observations using the $2.12 \mathrm{~m}$ telescope located at OAN SPM, with the low to intermediate resolution Boller \& Chivens (B\&Ch) spectrograph. We acquired spectra with a resolution of $5.5 \AA$ using a 600 lines $/ \mathrm{mm}$ grating to cover a $4300-5700 \AA$ range. The observations were made through a $1.5^{\prime \prime}$ slit, oriented in the east-west direction. CuHeNeAr lamp exposures were taken every 60 min for wavelength calibration and for flux calibration; spectrophotometric standards from Oke (1990) catalog were observed. The exposure time was $900 \mathrm{~s}$ per spectrum. The image processing was carried out with standard IRAF procedures (Tody 1986, 1993).

\section{DATA ANALYSIS AND RESULTS}

\subsection{Light Curve Morphology}

We observed CV SDSSJ 122405.58+184102.7 over ten nights, spread over two years, covering $42 \mathrm{hr}$ of total photometry. The system was at quiescence during our observations, as we can see in the top panel of Figure 1. However, individual light curves (individual 10 frames in Figure 1) have two conspicuous brightness variations, occurring at two different time scales but with almost the same amplitude: a long photometric signal with $\approx 0.2$ mag amplitude and a short one with $\approx 0.1 \mathrm{mag}$ amplitude.

On HJD $=836$ (frame N4 on Figure 1) the light curve exhibits a sinusoidal pattern with a mean amplitude of $\approx 0.1 \mathrm{mag}$ and a period of tens of minutes. We can appreciate the later in more detail in the upper panel of Figure 2. However, this modulation nearly disappears when the observation time is longer, like in HJD = 837 (frame N5 in Figure 1). In the bottom panel of Figure 2 the long term photometric signal is highlighted. In that case, the brightness changes mainly because of the orbital motion. Even masked inside the long photometrical signal, the $0.1 \mathrm{mag}$ modulation is present on other nights (darker circles in Figure 1).

\subsection{Photometric Orbital Period}

In Figure 1, all observations are displayed in different frames. We applied a discrete Fourier transform (DFT) algorithm, using the software Period04 (Lenz \& Breger 2005), to our complete photometric data set to search for significant periodic signals. The results obtained in this way are compatible with a Lomb-Scargle periodogram.

Panel (a) of Figure 3 shows a fraction of the total power spectrum for a ten days light curve after subtracting the spectral window. We explored the frequency range from 0 to $600 \mathrm{cy}-$ cles day $^{-1}$ (the Nyquist limit). However, the power spectrum is dominated by short frequencies. The maximum frequency is located at $\Omega=$ 5.959119 cycles day ${ }^{-1}$ with a SNR of 7.98.

According to empirical results of Breger et al. (1993), and numerical simulations from Kuschnig et al. (1997), a power spectrum with a SNR larger than 4.0 is needed to ensure that the signal is a real feature. We carried out a Monte Carlo simulation to improve the orbital frequency and to calculate its uncertainty. This kind of Monte Carlo simulation is described in Mennickent \& Tappert (2001), Mennickent et al. (2002) and Aviles et al. (2018). We generated 1000 data sets with the same HJD as the original time string and the magnitudes were those obtained by the last fit plus Gaussian noise. For every new data set, a least-squares fit was computed. The frequency distribution of this simulation was used to improve the orbital frequency and the uncertainty corresponds to the standard deviation of this distribution. With this procedure, the frequency was improved to 5.959076 cycles day $^{-1}$ which corresponds to a period $P=1 / \Omega=0.167811(1)$ days $(=4.027464(3) \mathrm{h})$ adopted here as the orbital one. In panel (b) of Figure 3 we present a Monte Carlo histogram that shows the frequency improvement compared with that obtained from the FFT power spectrum (inset). Finally, in panel (c) of Figure 3 we show the folded light curve with the period $P=4.03 \mathrm{~h}$ beside a sinusoidal fit to remark the 0.2 magnitude long term modulation. 


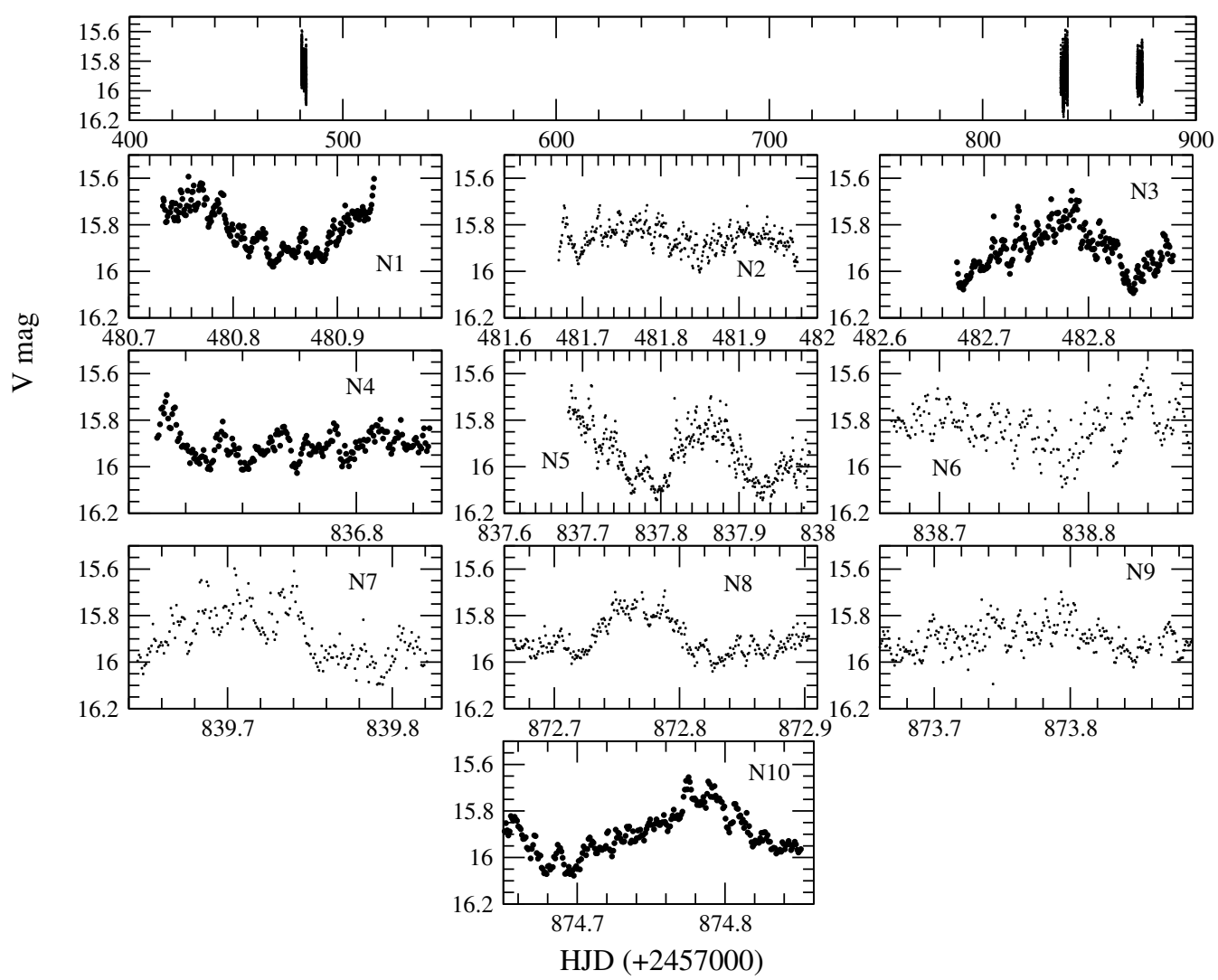

Fig. 1. The top panel shows the light curve ( $V$ magnitude as function of time) for SDSSJ1224 acquired over two years. The system was always at its quiescence state during observations $(\approx 15.9$ magnitudes $)$. In the bottom panels we show individual light curves for each observation night. We identify two brightness variations occurring at different time scales. This is evident by eye inspection in the panels labeled N4 and N5.

\subsection{Short Time-Scale Variability in the Light Curve}

The power spectrum is the most widely used tool to detect any quasi-periodic oscillation. In such a case, the power spectrum will show a broad peak that covers many frequencies, rather than a narrow peak centered on a given frequency. In other words, these oscillations are not coherent over time. They can change in a time scale of less than a day, like in V442 Oph and RX J1643.7+3402 (Patterson et al. 2002 ), or can be coherent over 20 cycles like in HS 0728+6738 (Rodríguez-Gil et al. 2004).

To inquire if a short time-scale oscillation is present in other nights, and to gain insight about the HDJ $=836$ light curve behavior, we used Period 04 to analyze the data marked with darker circles in Figure 1 (Frames N1, N3, N4 and N10). We selected at least one night from each observational campaign. In some cases it was necessary to detrend the data to be able to carry out the period analysis, as in Kennedy et al. (2016), where they masked the light curve to remove the eclipses in
MASTER OTJ192328.22+612413.5. We explored a wide range of frequencies as was done for the orbital period analysis. In Panel (a) of Figure 4 we show a portion of the power spectrum, and the frequency that modulates this data with a high probability $\omega=50.423395$ day $^{-1}$, equivalent to a period of $P=28.558 \mathrm{~min}$. We also carried out a Monte Carlo simulation to improve this value. The histogram in Panel (b) of Figure 4 shows the most favored frequency for this variability, $\omega=50.423990$ days $^{-1}$. In Panel (c) we present the data folded with a period of 28.6 min, where a wave pattern is observed with an amplitude of $\approx 0.1$ magnitudes.

We assume that this short-periodic variation may be related to the spin period of the WD. Therefore, SDSSJ1224 could be classified as an IP. Another evidence in favor of an IP nature is the detection of beat frequencies $\omega-2 \Omega, \omega-\Omega, \omega+\Omega, \omega+2 \Omega$, where $\omega$ and $\Omega$ are spin and orbital frequencies, respectively. The existence of the spin-orbital sidebands for IPs was pointed out by Warner (1986) and Wynn \& King 

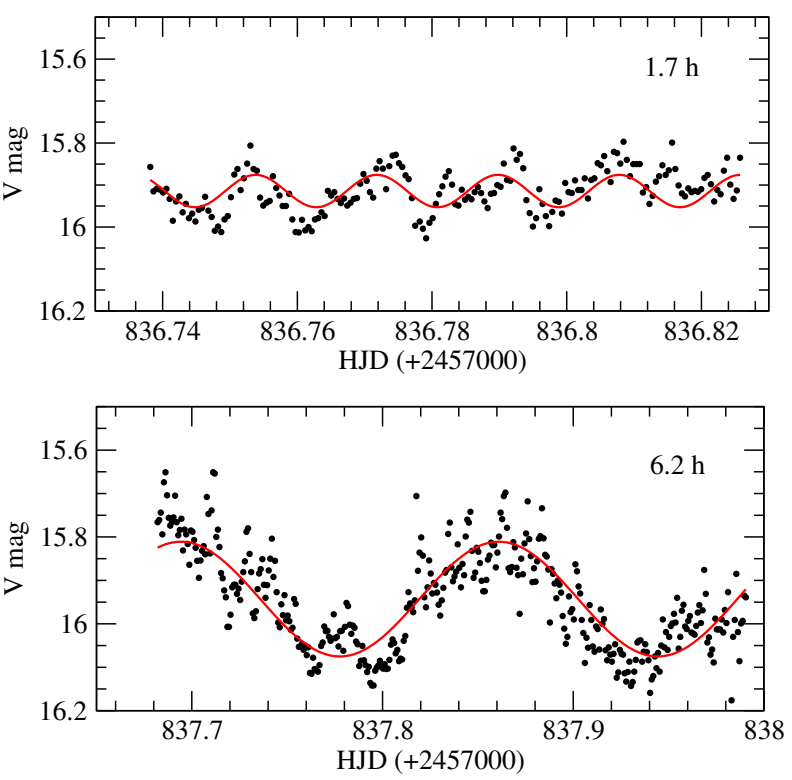

Fig. 2. Two time-scales for the two brightness variations. Upper panel, light curve for HJD $=836$ showing the short photometric signal with $\approx 0.1 \mathrm{mag}$ amplitude. Bottom panel, light curve for HJD $=837$ showing the long photometric signal with $\approx 0.2$ mag amplitude. The observation time span is indicated at each panel. The color figure can be viewed online.

(1992). The optical beat period is thought to arise from the reprocessing of soft X-rays by parts of the system fixed on the binary frame, like the secondary star itself.

\subsection{Spectroscopic Features of SDSSJ1224}

The average of 36 spectra of SDSSJ1224, acquired on March 19, 2017 (HJD $\approx$ 834.66), is presented in Figure 5. This spectrum shows single-peaked line profiles dominated by hydrogen (Balmer series) and HeI $(\lambda 5015, \lambda 4922$ and $\lambda 4388)$ emission lines. High excitation lines like HeII $\lambda 4886$, the CIII / NIII $\lambda 4645$ Bowen blend and OII $\lambda 4416$ are also present. Of particular interest is the presence of the HeII $\lambda 4886$ line, because it is recognized as evidence of the presence of a source of ionizing photons, typical of magnetic CVs or NL variables (Araujo-Betancor et al. 2003; Rodríguez-Gil et al. 2004). Table 2 provides the equivalent widths (EW) and the full-width halfmaximum (FWHM) of the main emission lines detected in the averaged spectrum. The FWHMs were obtained by fitting a single Gaussian to the line profiles. Taking into account the small FWHM of the lines, the inclination angle is expected to be low for SDSSJ1224.

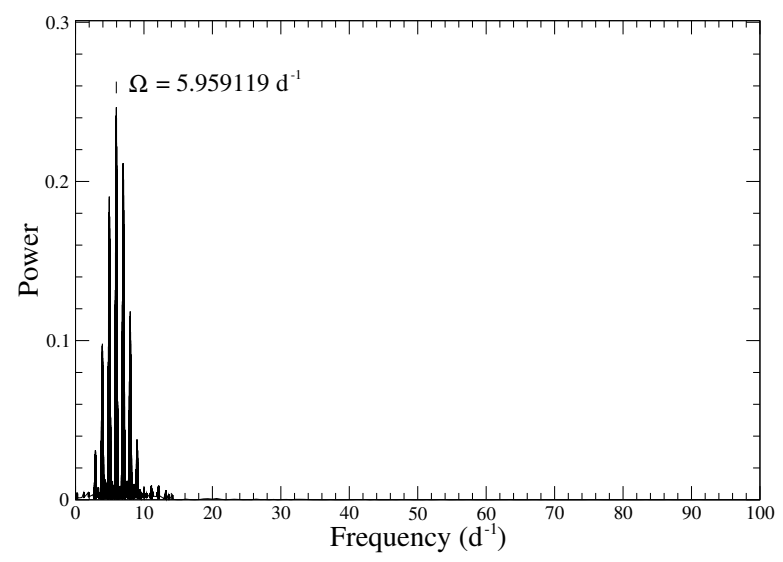

(a) Power Spectrum

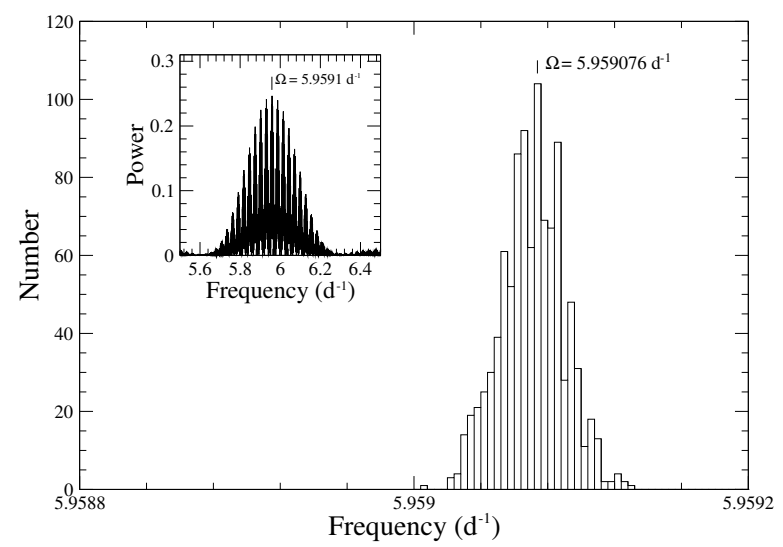

(b) Monte Carlo histogram

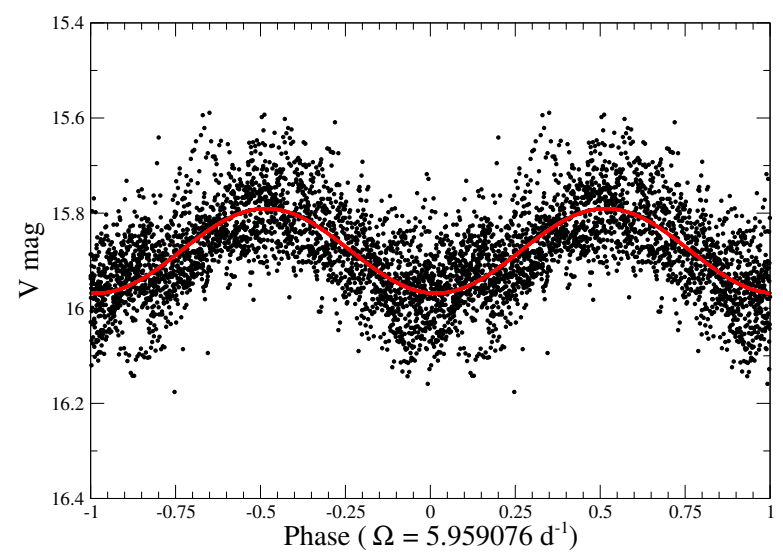

(c) Folded light curve

Fig. 3. (a) Power spectrum for the 10-days light curve after subtracting the spectral window, indicating the most probably frequency. (b) Histogram generated from the Monte Carlo simulation, showing an improved frequency in our data set. (c) Phase-folded light curve and leastsquares fit, shown with a red solid line (see the online version); all data were fit to a period of $P=1 / \Omega$. The color figure can be viewed online. 


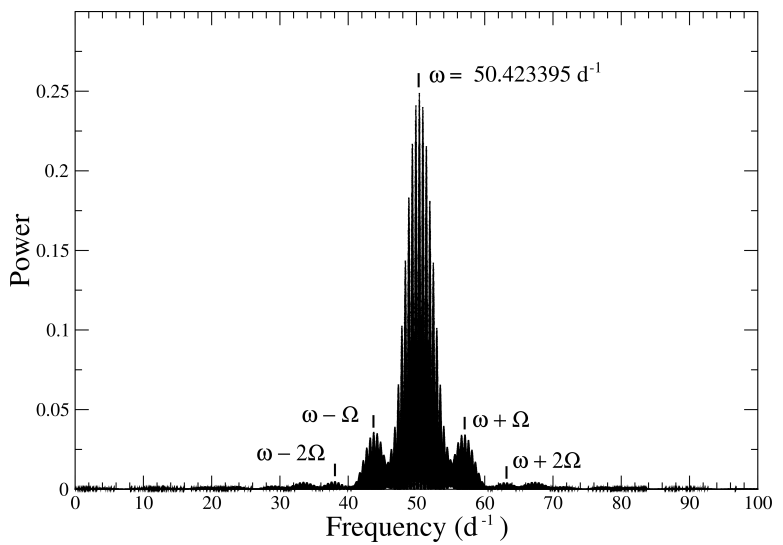

(a) Power Spectrum

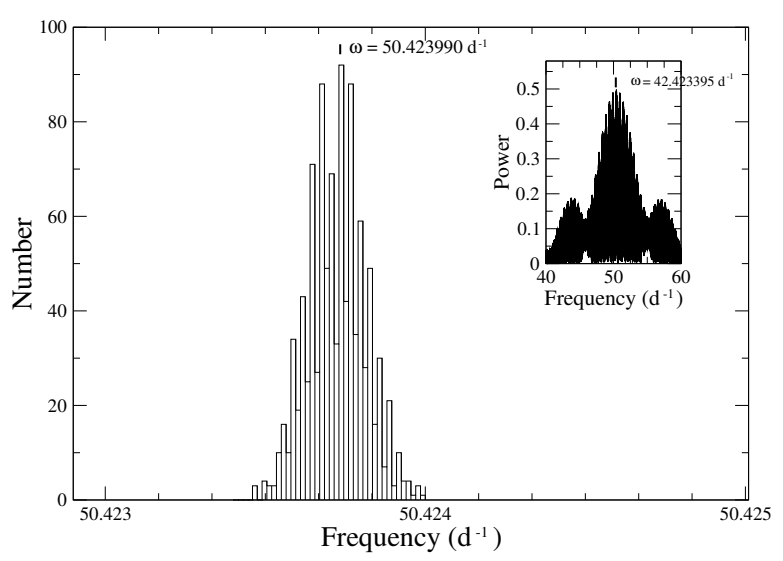

(b) Monte Carlo histogram

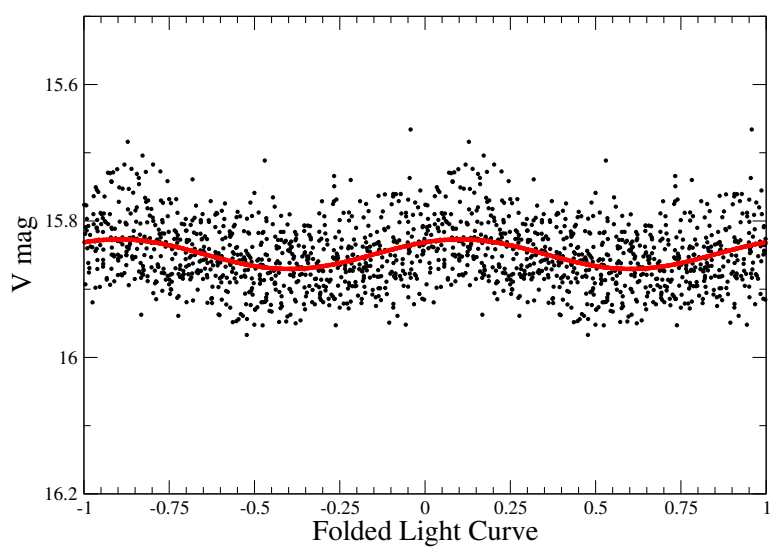

(c) Folded light curve

Fig. 4. (a) Power spectrum for the data marked with open circles in Figure 1, the frequency is $\omega=$ 50.423395 day $^{-1}$. (b) Histogram generated from the Monte Carlo simulation, showing an improved spin frequency. (c) Folded data with the frequency for the short period variability $P=28.6 \mathrm{~min}$. The red solid line (see the online version) is a least-squares fit to the data. The color figure can be viewed online.
TABLE 2

SDSSJ1224 EMISSION LINES: MARCH 2017

\begin{tabular}{lcc}
\hline \multicolumn{1}{c}{ Line } & FWHM $[\AA]$ & EW $[\AA]$ \\
\hline $\mathrm{H} \gamma$ & 6.425 & 1.312 \\
$\mathrm{He}$ II $\lambda 4886$ & 9.512 & 0.506 \\
$\mathrm{H} \beta$ & 7.177 & 2.035 \\
$\mathrm{He} \mathrm{I} \lambda 4922$ & 7.032 & 0.234 \\
$\mathrm{He} \mathrm{I} \lambda 5015$ & 8.850 & 0.256 \\
\hline
\end{tabular}

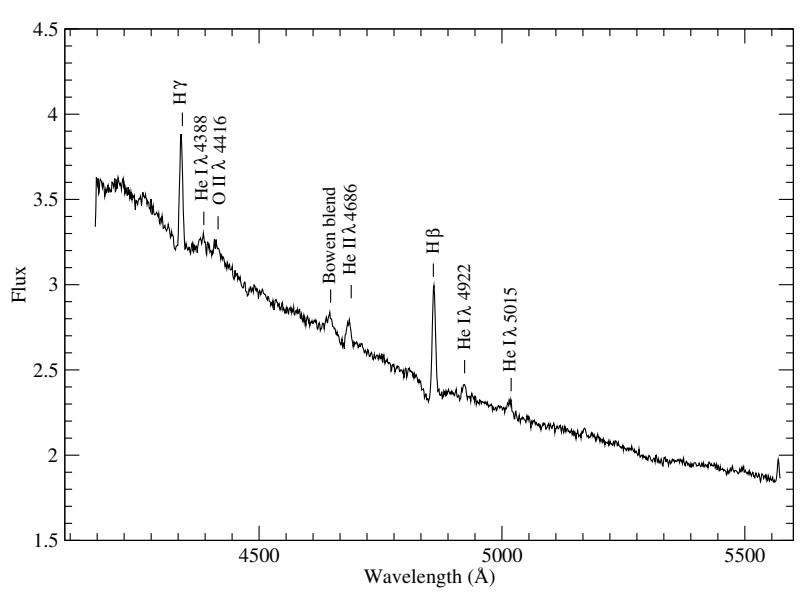

Fig. 5. Averaged flux calibrated spectrum of SDSSJ1224. The major lines are marked. Vertical axis is in units of flux density $F_{\lambda} \times 10^{-15} \mathrm{erg} \mathrm{cm}^{-2} \mathrm{~s}^{-1} \AA^{-1}$.

SDSSJ1224 has an optical spectrum in quiescence similar to RX J1643.7+3402, HS 0728+6738 and HS $1813+6122$, as reported by Patterson et al. (2002), Rodríguez-Gil et al. (2004), Rodríguez-Gil et al. (2007), respectively. In all cases, they argue the SW Sex membership according to spectroscopic properties and photometric variabilities. RodríguezGil et al. (2009) detected circular polarization in RX J1643.7+3402, confirming in this way the magnetic nature of SW Sex stars.

In Figure 5 we observe strong evidence for the detection of the WD as absorption in the blue wings of both the $\mathrm{H} \beta$ and $\mathrm{H} \gamma$ lines. This is in agreement with the results reported by Szkody et al. (2011, 2014). Our photometry and FWHM measurements support the idea of a long orbital period, as well as a low inclination angle for SDSSJ1224, instead of a low accretion rate as the reason for the WD detection.

\section{DISCUSSION}

CV SDSSJ1224 is a non-eclipsing binary system that has certain photometric features to potentially lead to a classification as a SW Sex star. First, we found 
an orbital period of $4.03 \mathrm{~h}$. According to RodríguezGil et al. (2007), the orbital periods for such systems are from 3 to $4.5 \mathrm{~h}$. Almost $50 \%$ of the CV population in this range has been classified as SW Sex stars. This sample includes eclipsing and non-eclipsing systems.

Second, we detected quasi-periodic oscillations with a period of 28.6 minutes. In terms of short period variabilities (kilo-seconds), SW Sex stars are also characterized by exhibiting quasi-periodic modulations in their light curves. In a compilation of CVs that show fast oscillations, Warner (2004) presented nine SW Sex stars that show quasi-periodic oscillations (QPOs) with a predominant time scale of $\approx 1000-2000 \mathrm{~s}(16.7-33.3 \mathrm{~min})$.

Third, another fact that supports our conjecture is that assuming an accretion disk-magnetic field interaction model, as proposed by Rodríguez-Gil et al. (2001), the spin period and the orbital one are related by

$$
P_{\text {spin }} \simeq 0.31 f^{3 / 2} P_{\text {orb }} .
$$

The previous equation results from considering that the shock between the gas stream and the accretion disk occurs close to the co-rotation radius. Here $f$ represents the co-rotation radius expressed in units of $R_{L 1}$ (the distance between the WD and the inner Lagrangian point, $\left.L_{1}\right)$. In the case of SDSSJ1224, the value for $f$ is 0.53 , which is in good agreement with values reported for the prototype SW Sex itself (Groot 1999); LS Peg (Rodríguez-Gil 2001); V533 Her (Rodríguez-Gil \& Martínez-Pais 2002). As a consequence of this model, SW Sex stars are indeed IP with the highest mass accretion rates.

Fourth, variabilities on time scales of minutes to tens of minutes have been detected in the optical light curve of several IPs (e.g., Patterson et al. 2002), and they are recognized as the spin period of the magnetic WD. We consider that, from its spectral appearance during low state (Figure 5) and the optical variability detected, SDSSJ1224 is indeed a new IP SW Sex class member. We suggest that the detected frequency of 50.423990 day $^{-1}$ is the WD spin frequency $\omega\left(P_{\text {spin }}=1 / \omega\right)$. The quasi-sinusoidal shape of its light curve, which is typical for the spin light curve of IPs (see e.g., the spin light curve of FO Aqr by de Martino et al. 1994), lends support to our hypothesis that this frequency is indeed the spin frequency of the WD.

Fifth, magnetic WDs in spin equilibrium should closely satisfy the ratio $P_{\text {spin }} / P_{\text {orb }}=0.1$ (King \& Lasota 1991), assuming that the accretion process is not primarily through a disk. The QPOs may reflect an underlying rotation of a magnetic WD (Patterson et al. 2002). In the case of SDSSJ1224 this assumption seems quite valid, since if the $28.6 \mathrm{~min}$ signal is the spin period of the WD, then $P_{\text {spin }}=0.12 P_{\text {orb }}$, which is very close to the value around which IPs seem to cluster on hard X-ray surveys (Scaringi et al. 2010).

\section{FINAL COMMENTS AND CONCLUSIONS}

We conclude that, based on the overall light curve features, the reported photometric periods and the spectroscopic properties, the system SDSSJ1224 can be classified as a new non-eclipsing SW Sex. The observed behavior matches all the conditions to be a member of this class of CVs.

It has a photometric orbital period of $4.03 \mathrm{~h}$, which lies in the range of periods where the vast majority of SW Sex stars are grouped. It shows photometric features common to this type of stars and, as such, the number of non-eclipsing systems of this particular type in this range of periods is increased.

We detect a brightness variation amplitude of 0.1 mag and a period of $28.6 \mathrm{~min}$, that we associate to the spin period of the WD. Also, we detect beat frequencies between the WD spin frequency, $\omega$, and the orbital frequency, $\Omega$, of the order of 30 minutes $\left(P_{\text {orb }}=8 P_{\text {beat }}\right)$ like in the system RX J1643.7+3402 (Rodríguez-Gil et.al. 2009).

The photometric behavior fits very well with those observed on the out-of-eclipse light curve of several CVs like HS 0728+6738 (Rodríguez-Gil et al. 2004) or MASTER OTJ192328.22+612413.5 (Kennedy et al. 2016). In particular, these two objects show a QPO amplitude of 0.2 mag with a period around 20 minutes, quite similar to SDSSJ1224. This result may imply that the QPOs are not exclusive to eclipsing systems, as was pointed out by Patterson et al. (2001).

Because quasi-periodic oscillations in the range of kilo-seconds have recently been detected in confirmed SW Sex stars (Patterson 2002; Kennedy et al. 2017; Rodríguez-Gil et al. 2007), we consider that SW Sex stars are actually members of IP CVs stars.

The physical conditions present in this type of binary systems are not well understood and should be addressed both theoretically and observationally. However, such a study is beyond the scope of this work.

This work is based upon observations carried out at the Observatorio Astronómico Nacional at the Sierra San Pedro Mártir (OAN-SPM), Baja California, México. We thank the daytime and night support staff at the OAN-SPM for facilitating and help- 
ing us to obtain the observations. The authors also acknowledge PAICyT-UANL grant CE642-18 for resources provided toward this research. We are also thankful to the anonymous referee for a careful reading of the manuscript and for useful comments that improved the content of this work.

\section{REFERENCES}

Araujo-Betancor, S., Gänsicke, B. T., Hagen, H.-J., et al. 2003, A\&A, 406, 213

Avilés, A., Chávez, C., García, P., et al. 2018, RMxAA, 54,389

Balman, Ş., Godon, P., \& Sion, E. M. 2014, ApJ, 794, 84

Breger, M., Stich, J., Garrido, R., et al. 1993, A\&A, 271, 482

Campbell, C. G. 1997, MNRAS, 291, 250.

de Martino, D., Buckley, D. A. H., Mouchet, M., et al. 1994, A\&A, 284, 125

Groot, P. J. 1999, Ph.D. Thesis

Hellier, C., Cropper, M., \& Mason, K. O. 1991, MNRAS, 248,233

Hoard, D. W., Szkody, P., Froning, C. S., et al. 2003, AJ, 126, 2473.

Howell, S. B., Szkody, P., \& Cannizzo, J. K. 1995, ApJ, 439, 337.

Kennedy, M. R., Callanan, P., Garnavich, P. M., et al. 2016, AJ, 152, 27

Kennedy, M. R., Callanan, P., Garnavich, P. M., et al. 2017, MNRAS, 466, 2202.

King A. R., Lasota J.-P., 1991, ApJ, 378, 674

Knigge, C. 2011, ASPC, 447, 3

Kuschnig, R., Weiss, W. W., Gruber, R., et al. 1997, A\&A, 328, 544

Lenz, P. \& Breger, M. 2005, CoAst, 146, 53.

Mennickent, R. E. \& Tappert, C. 2001, A\&A, 372, 563

Mennickent, R. E., Tappert, C., Gallardo, R., Duerbeck, H. W., \& Augusteijn, T. 2002, A\&A, 395, 557

Meyer, F. \& Meyer-Hofmeister, E. 1981, A\&A, 104, L10

Mizusawa, T., Merritt, J., Ballouz, R.-L., et al. 2010, PASP, 122, 299
Oke, J. B. 1990, AJ, 99, 1621

Paczynski, B. \& Sienkiewicz, R. 1983, ApJ, 268, 825

Patterson, J., McGraw, J. T., Coleman, L., et al. 1981, ApJ, 248, 1067

Patterson, J., Fenton, W. H., Thorstensen, J. R., et al. 2002, PASP, 114, 1364

Rappaport S., Verbunt F., \& Joss P. C. 1983, ApJ, 275, 713

Rodríguez-Gil, P., Casares, J., Martínez-Pais, I. G., et al. 2001, ApJ, 548, L49

Rodríguez-Gil, P., Gänsicke, B. T., Barwig, H., et al. 2004, A\&A, 424, 647

Rodríguez-Gil, P., Gänsicke, B. T., Hagen, H.-J., et al. 2007, MNRAS, 377, 1747

Rodríguez-Gil, P., \& Martínez-Pais, I. G. 2002, MNRAS, 337, 209

Rodríguez-Gil, P., Martínez-Pais, I. G., \& de la Cruz Rodríguez, J. 2009, MNRAS, 395, 973

Rodríguez-Gil, P., Schmidtobreick, L., \& Gänsicke, B. T. 2007, MNRAS, 374, 1359

Rosen, S. R., Mason, K. O., \& Cordova, F. A. 1988, MNRAS, 231, 549

Scaringi S., Bird, A. J., Norton, A. J., et al. 2010, MNRAS, 401, 2207

Schmidtobreick, L., Rodríguez-Gil, P., \& Gänsicke, B. T. 2012, MmSAI, 83, 610

Spruit, H. C., \& Ritter, H. 1983, A\&A, 124, 267

Szkody, P., Anderson, S. F., Brooks, K., et al. 2011, AJ, $142,181$.

Szkody, P., Everett, M. E., Howell, S. B., et al. 2014, AJ, 148, 63

Szkody, P. \& Mattei, J. A. 1984, PASP, 96, 988

Thorstensen, J. R., Ringwald, F. A., Wade, R. A., et al. 1991, AJ, 102, 272

Tody, D. 1986, Instrumentation in Astronomy VI, 733 1993, ASPC 52, Astronomical Data Analysis

Software and Systems II, ed. R. J. Hanisch, R. J. V.

Brissenden, \& J. Barnes, 173

Verbunt, F. \& Zwaan, C. 1981, A\&A, 100, L7

Warner, B. 1986, MNRAS, 219, 347

1995, Cataclysmic Variable Stars (CUP) 2004, PASP, 116, 115

Wynn, G. A. \& King, A. R. 1992, MNRAS, 255, 83

Héctor Aceves Campos: Instítuto de Astronomía, Universidad Nacional Autónoma de México, Apdo. Postal 106, Ensenada, Baja California, 22860, México (aceves@astro.unam.mx).

Andrés Alberto Avilés Alvarado and Carlos Esteban Chávez Pech: Facultad de Ingeniería Mecánica y Eléctrica, Universidad Autónoma de Nuevo León, Av. Universidad s/n Ciudad Universitaria San Nicolás de los Garza, Nuevo León, México, C.P. 66451 (andres.avileslv@uanl.edu.mx, carlosepech@yahoo.com).

Itzel Arias Rodríguez, José Enrique Pérez De León, Eduardo Gerardo Pérez-Tijerina, and Francisco Javier Tamayo Amaya: Facultad de Ciencias Físico Matemáticas, Universidad Autónoma de Nuevo León, Av. Universidad s/n Ciudad Universitaria San Nicolás de los Garza, Nuevo León, México, C.P. 66451 (pgtnuevoleon@hotmail.com, enrique.perezln@uanl.edu.mx, eduardo.pereztj@gmail.com, ftamayo@astrosen.unam.mx). 